\title{
Two-phase flow measurements under static and dynamic conditions for modeling the top kill process
}

\author{
M. Ghobadi • Y. S. Muzychka
}

Received: 3 April 2013/Accepted: 5 August 2014/Published online: 24 August 2014

(C) The Author(s) 2014. This article is published with open access at Springerlink.com

\begin{abstract}
An experimental study on bubble (slug) rise inside a pipe filled with static liquid and bull heading of a gas flow with a liquid flow is presented. The bubble rise velocity (BRV) was measured inside a pipe with different inclinations and is compared with two well-known models. The static tests have been performed with three different liquids: water, $\mathrm{CaCl}$ brine mixture, and non-Newtonian fluid (Xanvis ${ }^{\mathrm{TM}}$ ). A model is proposed, based on the drift flux method, to predict the bubble rise velocity. Dynamic well killing (or top kill) is also examined, whereby a continuous gas flow is suppressed with a counter current liquid flow. Flow tests at different angles from vertical were undertaken to investigate the effect of inclination on the liquid flow rate needed to bullhead the gas flow. A simple empirical model based on the results is proposed to predict the results in actual wells.
\end{abstract}

Keywords Bubble rise velocity - Bull heading . Two-phase flow C Counter current flow

\section{List of symbols}

\section{English symbols}

$C_{0} \quad$ Arbitrary constants

$C_{1} \quad$ Arbitrary constants

$C_{2} \quad$ Arbitrary constants

$D$ Diameter (m)

$g \quad$ Gravitational constant $9.81 \mathrm{~m} / \mathrm{s}^{2}$

$k_{1} \quad$ Constant

M. Ghobadi $(\bowtie) \cdot$ Y. S. Muzychka Faculty of Engineering and Applied Science, Memorial University of Newfoundland St. John's, Newfoundland A1B $3 \times 5$, Canada

e-mail:mehdi.ghobadi@mun.ca; y.s.muzychka@mun.ca $k_{2} \quad$ Constant

$N_{\mathrm{f}} \quad$ Inverse viscosity index

$Q \quad$ Volumetric flow rate $\left[(\mathrm{L} / \mathrm{s}),(\mathrm{SCFM}),\left(\mathrm{m}^{3} / \mathrm{s}\right)\right]$

\section{Greek Symbols}

$\theta \quad$ Pipe inclination off vertical

$\mu \quad$ Iscosity $\left(\mathrm{Ns} / \mathrm{m}^{2}\right)$

$\rho$ Density $\left(\mathrm{kg} / \mathrm{m}^{3}\right)$

$\sigma \quad$ Surface tension $(\mathrm{N} / \mathrm{m})$

\section{Dimensionless number}

Eo Eotvos number $\left(g D^{2}\left(\rho_{1}-\rho_{\mathrm{g}}\right) / \sigma\right)$

Fr Froude number $(U / \sqrt{g D})$

Re Reynolds number $(\rho U D / \mu)$

\section{Subscripts}

g Gas

1 Liquid

O Denotes vertical orientation

$\theta \quad$ Denotes at some angles off vertical

\section{Introduction}

A blowout is defined as an uncontrolled flow of formation fluids from a wellhead or wellbore. Although using the modern drilling equipment and appropriate crew training can reduce the risk, blowouts still occur. An appropriate well control procedure must be performed to remove the kick fluids and avoid additional formation fluid from flowing into the well (Vallejo-Arrieta 2002).

Gas may be released as individual bubbles or slugs when there is not enough flow to initiate a continuous stream of gas or a continuous stream when the gas flow is sufficient. Hence two conditions should be investigated to understand all blowout possibilities. In this study, the bubble rise

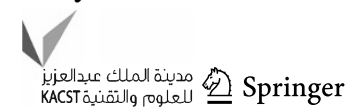


velocity (BRV) inside an inclined pipe and the liquid flow needed to bull-head a continuous stream of gas are investigated.

Bubble rise velocity under a static liquid column and dynamic killing of gas flow are examined experimentally using a multiphase flow loop with two different liquids: fresh water and $\mathrm{CaCl}$ (brine) mixture. The purpose of this paper was to provide a better understanding of bull-heading a producing gas well, by investigating the BRV and liquid flow rate needed to kill a flowing gas well.

Slug flows contain series of large and small bubbles characterized by long bullet-shaped bubbles, which are also called Taylor bubbles, and occupy nearly the entire cross section of the pipe. The liquid moves around the bubble or slug in a thin film and in the bulk flow between successive bubbles. The liquid flowing around the bubble expands at the rear of the bubble inducing a liquid wake. Slug flow is frequently encountered during the immiscible displacement of viscous oil in the porous rock of an oil-wet reservoir (Liao and Zhao 2003).

Dynamic behavior of the bubble rise in a liquid is the main concern of (Hua et al. 2009), while others (Berghmans 1973; Agarwal et al. 2010) examined the stability of the bubble inside the pipe. Numerical simulations (Hua et al. 2008) as well as experimental studies with particle image velocimetry (PIV) (Bugg et al. 1998, 2002; Sakakibara et al. 2007), have been conducted to understand the concepts of the bubble rise velocity.

A bubble or slug rises through a denser liquid because of its buoyancy. The experimental results of White and Beardmore (see Wallis 1969) provide an excellent summary of Taylor bubble terminal rise speeds. They identified the Froude number, Eotvos number, and Morton number as important dimensionless groups. Other studies have also used the Reynolds number and the Weber number as parameters (Bugg et al. 1998; Dziubinski et al. 2003; Wang et al. 2008; Han et al. 2010) and investigated the effects of liquid viscosity on bubble rise.

Fabre and Line (1992) reviewed the motion of Taylor bubbles in a larger review of slug flow. They mainly considered the case of zero liquid flow in vertical tubes. They stated that Taylor bubbles were characterized by leading edges shaped like prolate spheroids followed by a film on the wall of the tube and finally by a trailing edge. This trailing edge would be flat in cases where viscosity is unimportant and would be an oblate spheroid in cases where viscosity is important.

When viscous forces and surface tension forces are negligible, the Froude number is constant. The surface tension tends to reduce the Froude number. Bendiksen (1985) adjusted the Froude number for smaller Eotvos numbers (less than 40). They stated that the viscous regime is encountered for small inverse viscosity numbers.
However, for intermediate values (Eotvos number between 40-60) of the inverse viscosity number, no predictive methods for the Froude number are given.

Carew et al. (1995) proposed an empirical model for the effect of inclination on slug BRV. They examined the effect of increasing Newtonian fluid viscosity with reference to the momentum exchange and showed how viscosity reduces the inclination dependence of the Froude number. Their model is based on an integral momentum balance between stations far upstream and far downstream of the bubble nose which must balance the bubble's buoyancy in this region.

There are several well killing methods used to avoid the blowout in a live gas well. A subsea blowout can be brought under control by vertical re-entry into the blowout well by a rig positioned over the well, re-establishing pressure integrity of the wellhead/BOP by subsea means, drilling a relief well for a reservoir flood kill, or drilling a relief well for dynamic kill. Kastor and Letbetter (1974) published one of the first papers to discuss well killing which reviewed older methods such as constant bottomhole pressure method, which in turn infers maintaining a constant pump speed and for the largest part of the kill procedure, maintaining a constant drill pipe pressure.

The wait and weight method is a constant bottom hole pressure (BHP) method of circulating the kick out while pumping a heavier kill mud into the well to replace the original mud which was in the well when the kick occurred. The reservoir flood kill method requires drilling a relief well parallel to and near the blowout wellbore. Seawater is injected into the reservoir to reduce formation productivity and gradually kill the damaged wellbore. Another old way to kill the blowout well through relief well is called dynamic killing that has been studied by Koedtritz et al. (2008) and Rubiandini (2008), which involves injecting fluid from a relief well to the blowout well, usually water or high density fluid, to make the pressure in the blowout well exceed formation pressure so fluid flow will be stopped.

Additional case studies have been done. Adams et al. (2008) studied the model of offset subsea capping. Success of the methods for subsea well capping proved that safe and cost-effective work is possible adjacent to a burning blowout. Fram (1994) reviewed previous well killing methods and the damage each one imparted on the field and finally recommended a kill procedure for well under study. Salehi et al. (2008) studied the case where tectonic movements caused a sheared failure. Water and cement were pumped into the well and it was not successful. They completed the well kill by drilling two relief wells.

To kill a live closed-in gas well by bull-heading down the tubing, the selected pump rate should be high enough to ensure efficient displacement of the gas into the formation 
(i.e. to avoid the kill fluid bypassing the gas). On the other hand, the pressures that develop during bull-heading at a high rate must not exceed wellhead pressure rating, tubing or casing burst pressures or the formation breakdown gradient, since this will lead at best to a very inefficient job. Given these constraints, the optimum kill rate, required hydraulic horsepower, density, and type of kill fluids have to be selected.

Bull-heading of a live gas well was simulated in this paper, and the flow rate of the fluid needed to kill the gas flow inside the pipe was examined. We also measured the gas flow rate which kills the liquid flow in the beginning of each experiment. A general model is proposed for different flow rates and angles of the well. We measured the BRV through fresh water and a brine mixture inside the test pipe at different pipe inclinations. The Froude number is chosen to represent the data for these tests. We started our measurements using a vertical pipe and increased the angle by $15^{\circ}$ increments up to $75^{\circ}$. The results for these tests are compared to models from Wallis (1969) and Carew et al. (1995) for the inclination effect on the static bubble rise velocity.

\section{Simple theory}

Bubble rise experiments in vertical and inclined pipes have been considered by many researchers in the past. Much of the classical work on bubble rise velocity is found in the book by Wallis (1969) and the newer text by Levy (1999). It is now well established that three parameters influence the bubble rise velocity. The principal non-dimensional groups are balances between buoyancy and liquid inertia, liquid viscosity, or surface tension. When only one of these balances dominates, the BRV can be found to be a simple function as follows.

If the forces are fully dominated by inertial forces and buoyancy forces within the liquid, the balance can be shown to yield

$U_{0}=k_{1} \frac{\sqrt{g D\left(\rho_{1}-\rho_{\mathrm{g}}\right)}}{\sqrt{\rho_{1}}} \approx k_{1} \sqrt{g D}$

When liquid density is much greater than gas density (air versus water), the expression simplifies. If presented in non-dimensional form

$F r=\frac{U_{0}}{\sqrt{g D}} \approx k_{1}$,

where $F r$ is the Froude number. The value of the constant $k_{1}$ has been found experimentally to be $k_{1}=0.345$ (Wallis 1969) for vertical pipes.
If the forces are fully dominated by liquid viscosity and buoyancy forces, the balance yields

$U_{0}=k_{2} \frac{g D^{2}\left(\rho_{1}-\rho_{\mathrm{g}}\right)}{\mu_{1}}$,

where $k_{2}$ has been found to be $k_{2}=0.010$ (Wallis 1969) for vertical pipes.

The inverse viscosity number $N_{\mathrm{f}}$ is defined as

$N_{\mathrm{f}}=\frac{\sqrt{g D^{3}\left(\rho_{1}-\rho_{\mathrm{g}}\right) \rho_{1}}}{\mu_{1}} \approx \frac{\rho_{1} \sqrt{g D} D}{\mu_{1}}$

When liquid density is much greater than gas density, Eq. (4) simplifies as shown. One can see that $N_{\mathrm{f}}$ is similar to a Reynolds number if $(g D)^{1 / 2}$ is assumed to be a characteristic velocity. Therefore, if $N_{\mathrm{f}}$ is large, then the flow is inertially dominant. For water at standard conditions in our three-inch pipe tests, this gives $N_{\mathrm{f}}=66,150$, thus making our tests inertially dominant (Wallis 1969).

If surface tension is factor, then another group, the Eotvos number (also called the Bond number $B o=E o / 4$ ), becomes important. This is defined as follows:

$E o=\frac{g D^{2}\left(\rho_{1}-\rho_{\mathrm{g}}\right)}{\sigma}$

If surface tension dominates then the bubble is held statically in a column of liquid. This occurs when Eo $<3.37$ or Eo $\sim 1$ or less. The Eotvos effect is primarily responsible for bubble dynamics in small diameter tubes, i.e. those on the order of mm for water. For our water tests $E o=781$.

In general the BRV is a function of all variables such that

$k_{1}=f\left(N_{\mathrm{f}}, E o\right)$

or

$F r=k_{\mathrm{l}}\left(N_{\mathrm{f}}, E o\right)$

If the pipe is inclined, then this functional behavior is extended to include the inclination angle as well:

$k_{1}=f\left(N_{\mathrm{f}}\right.$, Eo,,$\left.\theta\right)$

or

$F r=k_{\mathrm{l}}\left(N_{\mathrm{f}}, E o, \theta\right)$

It has been found (Wallis 1969) that the BRV is inertially dominant when $N_{\mathrm{f}}>300$ and $E o>100$ and thus for a vertical pipe the BRV is

$k_{1}=0.345$

The BRV is viscosity dominant when $N_{\mathrm{f}}<2$ and Eo $>100$ and surface tension dominant when $E o<3.37$. If the viscosity number is large a simple model from Wallis (1969) is 
$k_{1}=0.345\left(1-\mathrm{e}^{(3.37-E o / 10}\right)$

This governs the BRV for smaller diameter pipes where surface tension effects become important.

In the case of inclined pipes we often define the normalized Froude parameter such that

$\frac{F r_{\theta}}{F r_{0}}=\frac{U_{\theta} / \sqrt{g D}}{U_{0} / \sqrt{g D}}=\frac{U_{\theta}}{U_{0}}=\frac{Q_{\theta}}{Q_{0}}=f\left(\theta, E o, N_{\mathrm{f}}\right)$,

where $Q$ is the volumetric flow rate. Depending on the balance of dominant forces this inclination function may be independent of the $E o$ and $N_{\mathrm{f}}$ numbers. Thus, if we conduct tests at variable inclinations, the normalized BRV curves are the inclination function for the given fluid, i.e. depend on properties but can be fit for that specific fluid. It is typical for a BRV to become maximum at an inclination angle of $45^{\circ}-60^{\circ}$ off vertical, then typically decrease after that. Most data in the literature are for inclinations less than $85^{\circ}$ from vertical.

We will present both static bubble rise and dynamic (kill) data using the normalized procedures and develop simple predictive formulas for interpolation purposes.

\section{Experimental facilities}

The flow measurements were conducted using the multiphase flow loop at Memorial University's Faculty of Engineering (Fig. 1). The flow loop has a capability of delivering up to $750 \mathrm{~L} / \mathrm{min}(\sim 200 \mathrm{gpm})$ of liquid flow. Compressed air can be injected either concurrently or counter currently. The flow loop is constructed from 3 inch PVC piping with clear sections for flow visualization capabilities. The original flow loop only allowed for vertical up, vertical down, and horizontal flow. The facility allows angles of $0,15,30,45,60$, and $75^{\circ}$ off vertical to be tested. Figure 2 shows the bubble rising in the pipe with $45^{\circ}$ inclination.

During the first series of tests, two fluids were examined at various inclination angles: Water and a Xanvis based gelled water solution. Xanvis is a liquid slurry containing $43 \%$ by weight of a highly refined, clarified, completion grade xanthan biopolymer in a dipropylene glycol methyl ether base. Xanvis is a premium quality xanthan product recommended for use where formation protection, solids suspension, and improved hole cleaning are primary concerns. Tests were conducted on the two fluids for two gas flow rates at the six prescribed inclination angles. Additional tests were also conducted on water in the vertical orientation to examine the effect of gas flow rate.

The first series tests showed that the dynamic kill velocity and static BRV are both similar functions of inclination angle, with the maximum liquid flow to kill a flowing gas wellbeing maximum at an inclination angle around $60^{\circ}$. The dynamic tests with the Xanvis based gelled water solutions resulted in a peculiar observation that no maximum occurred in the data. This was attributed to the non-Newtonian shear thinning nature of the fluid. A simple model fit was proposed for the water tests, but it was decided to retest using both water and a $\mathrm{CaCl}$ mixture. Further tests were also proposed including a static BRV test for each fluid as a function of inclination.

During the second series of tests, two fluids were once again tested. This time water was repeated along with tests using a $\mathrm{CaCl}$ Brine solution which was approximately $30 \% \mathrm{CaCl}$ by volume. Fluid properties for the $\mathrm{CaCl}$ Brine were obtained from a report Conde (2009). Tests for both fluids were conducted at all angles for 50 SCFM gas flow and again in the vertical orientation for $25,50,75$, and 100 SCFM gas flows. During all of our tests, the gas pressure
Fig. 1 Flow loop schematic of MUN multiphase flow loop

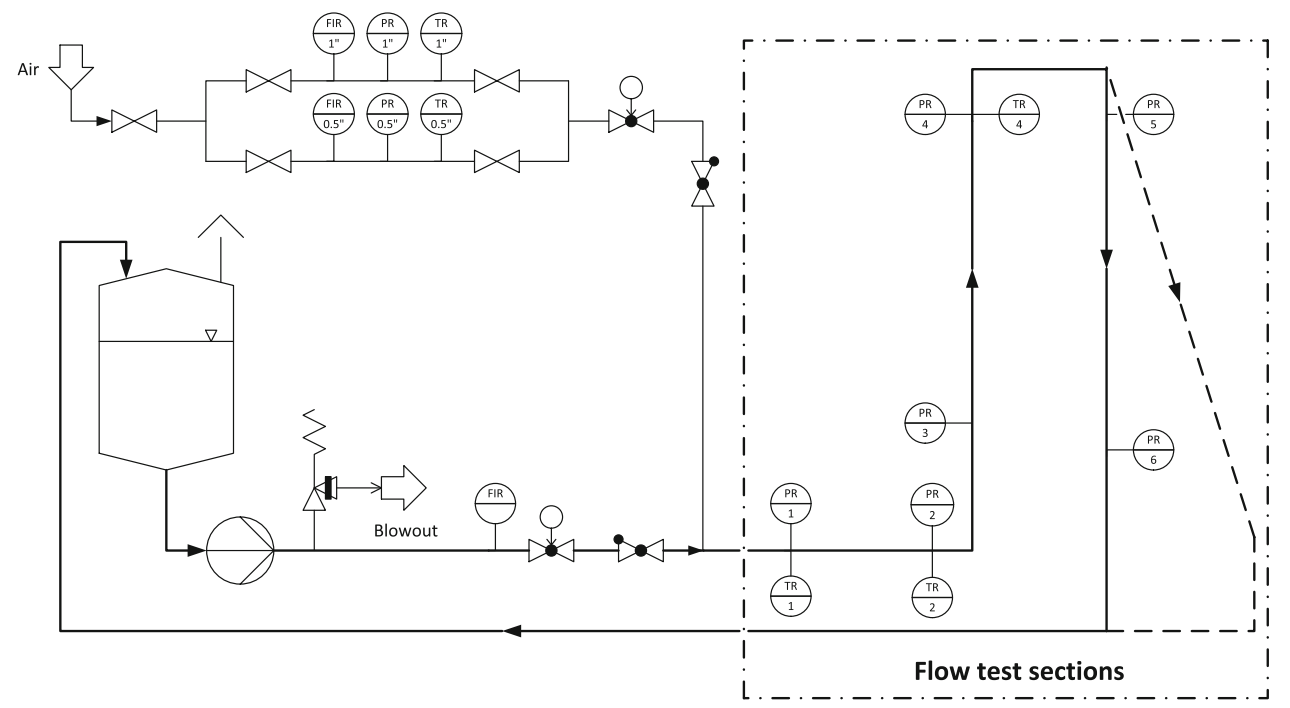




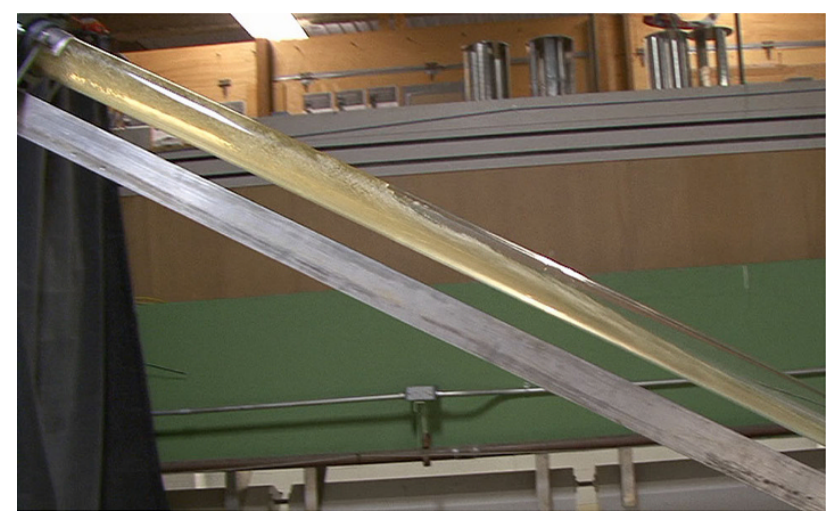

Fig. 2 Dynamic well kill simulation, (upward flowing gas), and (downward flowing liquid)

and temperature remained nearly constant. This gave an actual gas flow of 22.8 ACFM \pm 1.5 ACFM corresponding to the nominal 50 SCFM used for all inclined tests. A second set of tests were also conducted to determine the static bubble rise velocity at various inclinations and compared with theories in the two phase flow literature.

\section{Experimental procedures}

The general procedure for determining static BRV was to pre charge the gas lines with a finite volume of compressed gas. This gas was then released into the test column at various angles of inclination and the bubble rise speed was measured. This was achieved by means of two sensors. We used a pressure sensor at the base of the test section to measure gas flow pressure during dynamic tests. This sensor provided the instant that the gas bubbles passed this point. A thermal sensor was placed at the top of the test section and it recorded a decrease in temperature (relative to the water temperature) when the gas bubble passed. These sensor perturbations were recorded using a data logger, which also provided a time stamp for each reading. The maximum pressure and minimum temperature were chosen as the start and finish points and using the distance between sensors the BRV was easily measured. Tests were repeated approximately 15-20 times for each angle and the resulting data set were averaged to get the mean value for the given angle. The standard deviation for these tests varied over a small range, between 0.22 and $0.32 \mathrm{~m} / \mathrm{s}$, depending on angle of inclination.

In the case of dynamic kill data, the gas flow rate was pre-selected and set to a specific value of 50 SCFM ( 22.8 ACFM). With the flow loop set for a high liquid flow rate, the gas was observed to be directed through the return feed line for the two phase flow with no gas flowing upwards through the test section. Liquid flow rate was then gradually reduced until the gas flow became dominant and eventually killed the liquid flow while the pump continued to operate in a primed state. This liquid kill rate was recorded easily, as the dynamic data collection would show an abrupt decrease in liquid flow. It was found that this dynamic condition was almost independent of pipe inclination. Once the gas had killed the liquid flow, we gradually increased the liquid flow until the gas flow was bullheaded. Again, this condition was determined from the experimental data collected. Tests were repeated between 15 and 20 times at each angle of inclination until a smooth curve representative of the test was complete. The standard deviation for these tests varied over a larger range, between 5.5 and $10.37 \mathrm{~L} / \mathrm{min}$, depending on angle of inclination.

\section{Results and discussion}

\section{Static bubble rise results}

Results for the static bubble rise are depicted below in graphical form. We also present the normalized static bubble rise velocity, as effects of inclination and fluid properties can be objectively observed. Figures 3 and 4 summarize the static BRV tests in water and $\mathrm{CaCl}$ brine mixture. These tests provide a good baseline in assessing the effect of increased density, viscosity, and surface tension that the brine solution has on the simple static BRV. The brine mixture yielded approximately a $20 \%$ reduction on BRV as compared with water.

The normalized data shown in Fig. 4 are typical of data reported in the two-phase flow literature. Figures 3 and 4 illustrate the efficacy of the brine mixture on static BRV. The normalized plot shows that the higher the curve is, the lower the actual static BRV will be. Figures 5 and 6 show comparisons between the Carew et al. (1995) model, the Wallis (1969) model, and the data for water and brine mixtures. The general theory from Wallis (1969) provides

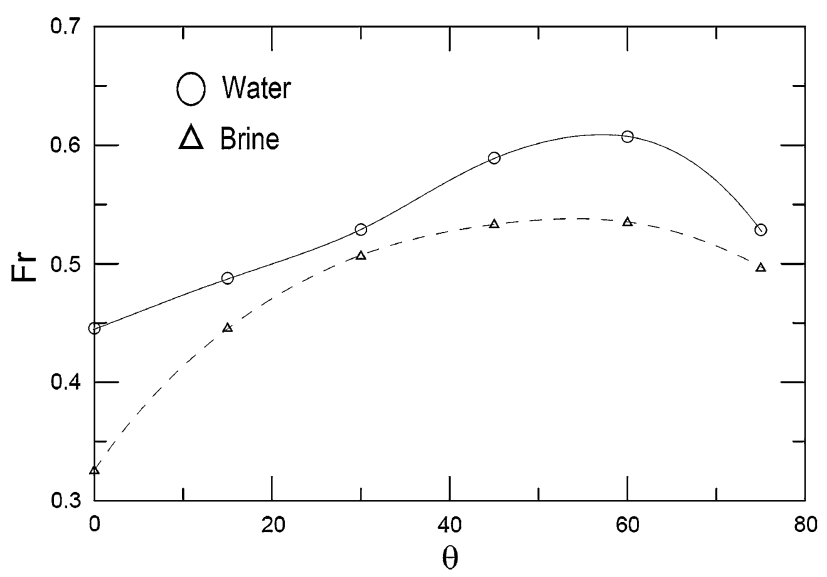

Fig. 3 Static bubble rise $F r=U /(g D)^{1 / 2}$ for water and brine mixture 


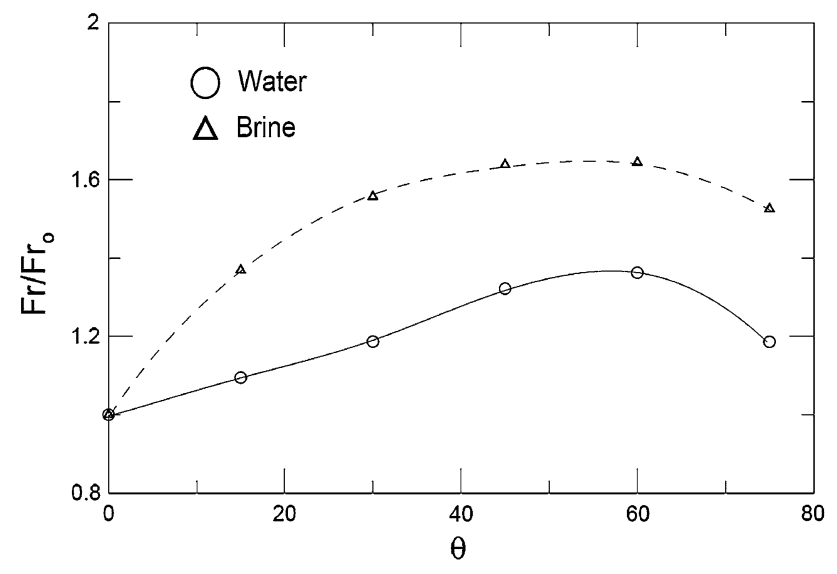

Fig. 4 Normalized static bubble rise $F r / F r_{0}=U / U_{0}$ for water and brine mixture

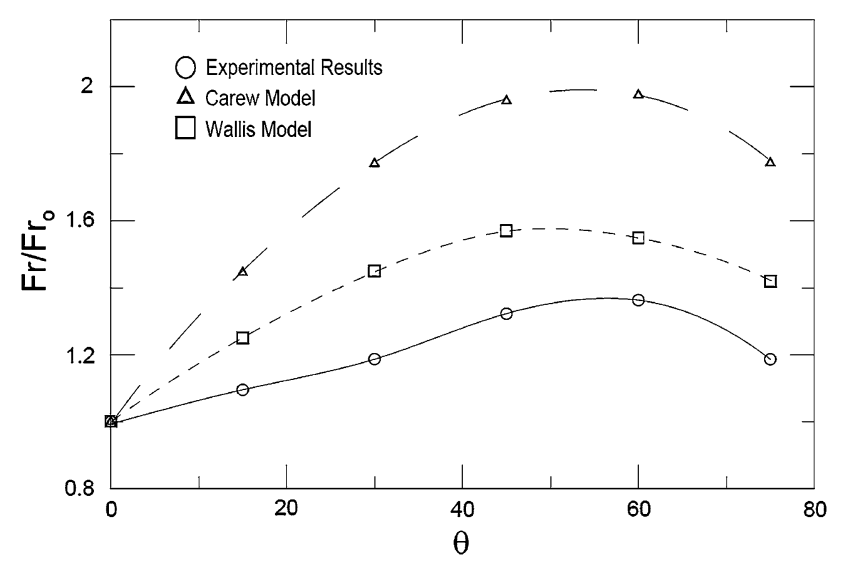

Fig. 5 Normalized static bubble rise, $F r / F r_{0}=U / U_{0}$ for water with predicted values

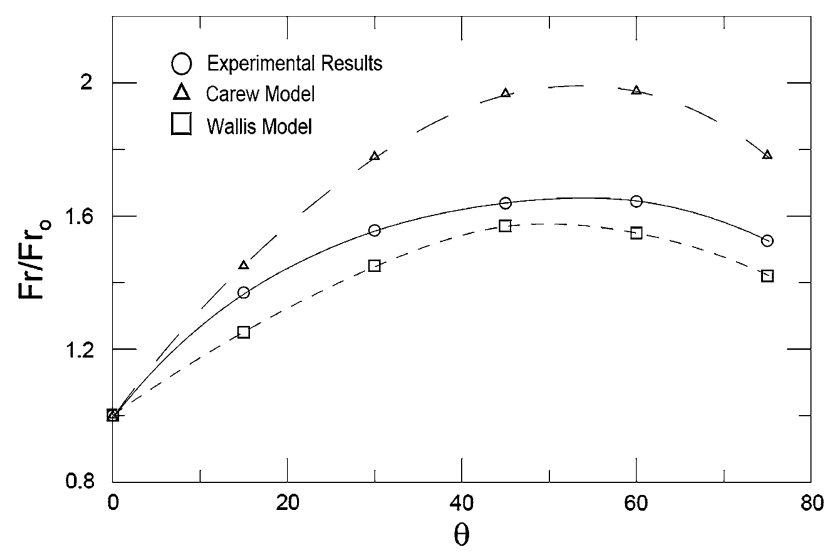

Fig. 6 Normalized static bubble rise, $F r / F r_{0}=U / U_{0}$ for brine with predicted values

good agreement with our present tests. The Carew et al. (1995) model seriously over predicts the present data. However, for the case of water, the equations should provide similar agreement with Wallis, as Carew's results

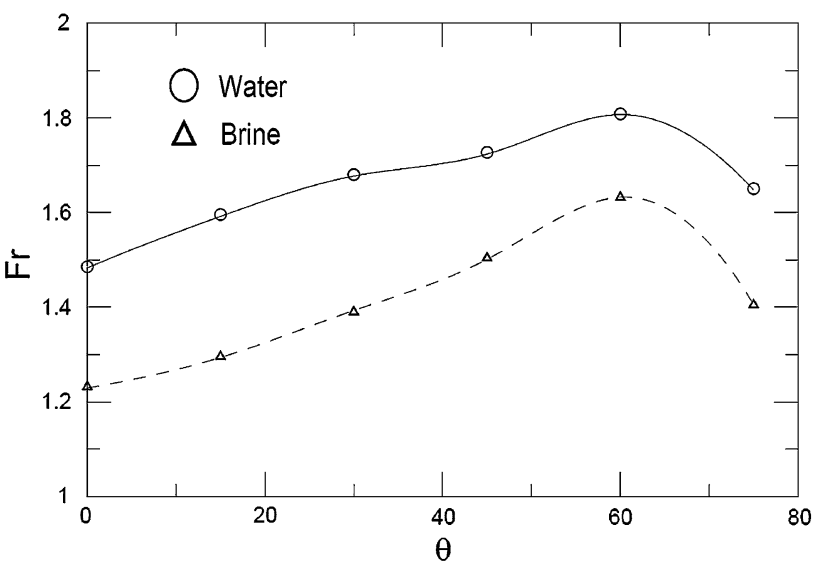

Fig. 7 Dynamic gas kill velocity, $F r=U /(g D)^{1 / 2}$ for water and brine mixture

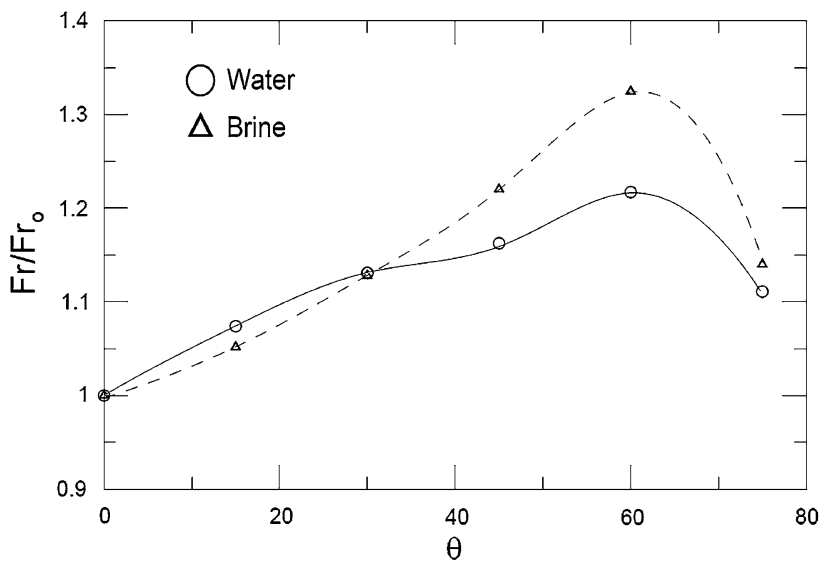

Fig. 8 Normalized dynamic gas kill velocity, $F r / F r_{0}=U / U_{0}$ for water and brine mixture

were also compared with Wallis's model. The model as reported in Carew et al. (1995) appears to have a mathematical error. Data in their paper are shown to agree with the model that they proposed, but for some unknown reason the reported equations do not produce the same curves shown in their paper.

Dynamic gas kill results

Results for the dynamic gas kill rates are given in Fig. 7 for both water and brine mixture when the gas flow is 50 SCFM. The normalized curves are also presented in Fig. 8 for both fluids. The normalized data show that the general behavior is almost the same as a function of inclination angle for both fluids, except for the region near maximum velocity, which occurs around the 55-65 inclination angles. The data for this gas flow rate were fit for each fluid to obtain a simple function of inclination angle. Further, we also conducted a series of tests for the vertical configuration whereby we varied the gas flow rate and measured the 
required liquid flow rate to bull-head kill the flowing gas. Tests were conducted at $25,50,75$, and 100 SCFM. It was decided that based on the first series tests, which show that the normalized inclination function is almost completely independent of gas flow, that only one set of test data at variable inclination was necessary. In earlier tests, the dynamic kill data were obtained for gas flow rates of 50 SCFM and 100 SCFM. Normalized curves for these results were in complete agreement with respect to the trend in inclination.

\section{Experimental data fits}

The normalized water data for variable inclination have been found to fit well with the following equation:

$$
\begin{aligned}
f_{\text {water }}(\theta)= & 1+0.051\left(\frac{\theta}{90}\right)+5.0757\left(\frac{\theta}{90}\right)^{2} \\
& -21.463\left(\frac{\theta}{90}\right)^{3}+34.145\left(\frac{\theta}{90}\right)^{4}-18.669\left(\frac{\theta}{90}\right)^{4},
\end{aligned}
$$

where the dynamic kill velocity is found using

$\frac{F r}{F r_{0}}=\frac{Q}{Q_{0}}=f_{\text {water }}(\theta)$

For the 50 SCFM gas flow, $F r_{0}=1.488$ for water. For the variable gas flow tests on the vertical pipe, the following behavior on gas kill flow rate for water was found:

$Q_{0}=5.328+0.0221 Q_{\mathrm{g}}$

The liquid flow rate is now given in $[\mathrm{L} / \mathrm{s}]$ and the gas flow rate in [standard L/s]. It is more appropriate to consider the actual superficial flow velocity of each phase (that is the velocity if the phase flowed alone in the pipe). Doing this, using actual gas flow rates yields for water

$U_{1}=1.1623+0.0484 U_{\mathrm{g}}$

This leads to the following predictive model for the water:

$U_{\text {water }}=\left[1.1623=0.0484 U_{\mathrm{g}}\right] \times f_{\text {water }}(\theta)$

Figure 8 depicts the dynamic kill predictions for water as the working fluid and five different gas speeds inside the test pipe.

The normalized brine data for variable inclination have been found to fit well with the following equation:

$$
\begin{aligned}
f_{\text {brine }}(\theta)= & -0.1369\left(\frac{\theta}{90}\right)+5.074\left(\frac{\theta}{90}\right)^{2} \\
& -19.130\left(\frac{\theta}{90}\right)^{3}+32.213\left(\frac{\theta}{90}\right)^{4}-19.244\left(\frac{\theta}{90}\right)^{4} \\
& -19.244\left(\frac{\theta}{90}\right)^{5}
\end{aligned}
$$

where the dynamic kill velocity is found using

$$
\frac{F r}{F r_{0}}=\frac{Q}{Q_{0}}=f_{\text {brine }}(\theta)
$$

For the $50 \mathrm{SCFM}$ gas flow, $F r_{0}=1.234$ for brine. For the variable gas flow tests on the vertical pipe, the following Correlation on gas kill flow rate for brine may be applied:

$Q_{0}=4.4586+0.0155 Q_{\mathrm{g}}$,

where the liquid flow rate is now given in $(\mathrm{L} / \mathrm{s})$ and the gas flow rate in (Standard L/s). It is more appropriate to consider the actual superficial flow velocity of each phase (that is the velocity if the phase flowed alone in the pipe). Doing this, using actual gas flow rates yields for brine

$U_{1}=0.9726+0.0340 U_{\mathrm{g}}$

This leads to the following predictive model for the brine mixture:

$U_{\text {brine }}=\left[0.9726+0.0340 U_{\mathrm{g}}\right] \times f_{\text {brine }}(\theta)$

Figure 9 depicts the dynamic kill predictions for brine as the working fluid and five different gas speeds inside the test pipe (Fig. 10).

\section{Predicting results for other pipe diameters}

In order to utilize the obtained data in a useful manner, simple fits were developed for each fluid. These fits are obtained in a piece wise manner with the inclination effect modeled first, followed by the effects of variable gas flow rate. The two fits were combined to obtain a simple model for predicting the dynamic gas kill liquid flow velocity as a function of inclination angle and actual gas flow velocity.

The Froude number is frequently used to scale the data for a desired pipe diameter D. All of our data were obtained in a nominal 3 in. diameter pipe. To appropriately scale the

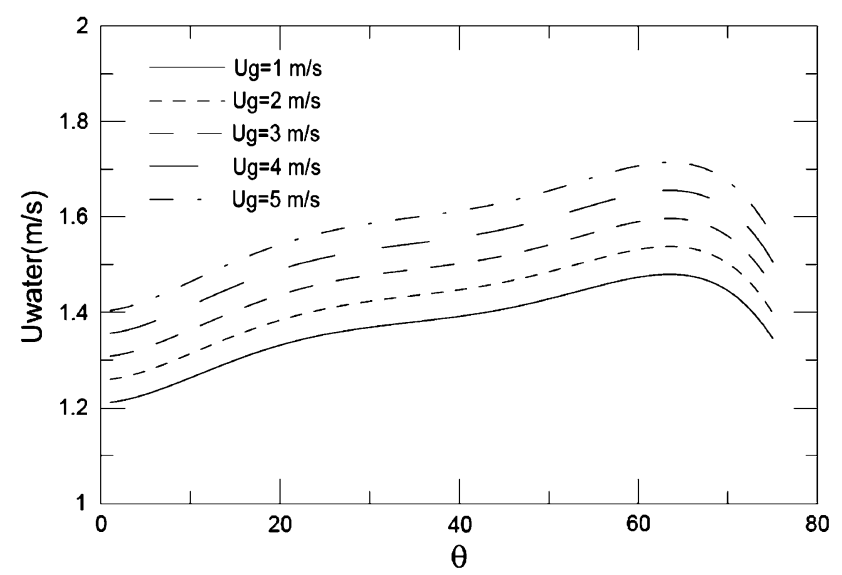

Fig. 9 Dynamic kill predictions for water for $U_{\text {gas }}=1,2,3,4$, and $5 \mathrm{~m} / \mathrm{s}$ for a 3 in. pipe 


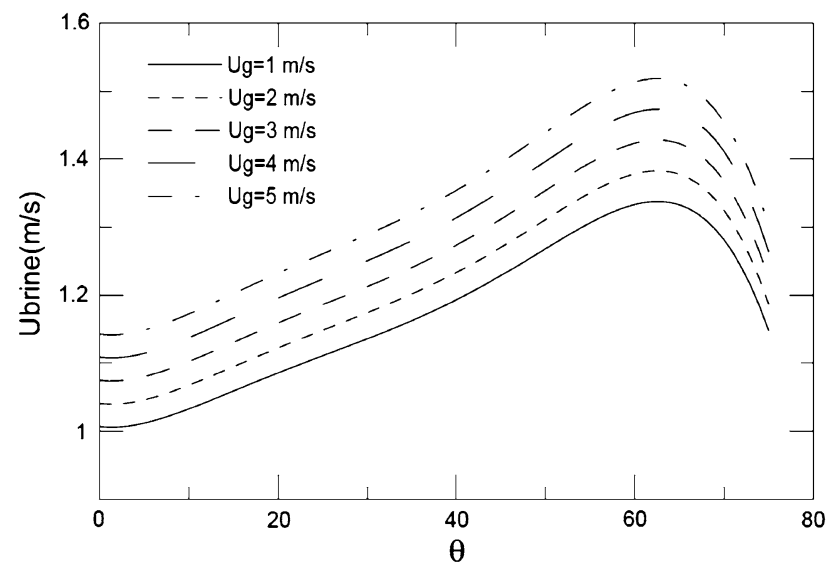

Fig. 10 Dynamic kill predictions for brine for $U_{\mathrm{gas}}=1,2,3$, 4, and $5 \mathrm{~m} / \mathrm{s}$ for a 3 in. pipe

data for larger pipes, Froude number scaling is used. Recalling that the Froude number is defined as

$F r=\frac{U}{\sqrt{g D\left(\frac{\rho_{1}-\rho_{\mathrm{g}}}{\rho_{1}}\right)}}$

One may define the following expressions from the developed fits:

$\frac{U_{3}}{\sqrt{g D_{3}\left(\frac{\rho_{1}-\rho_{\mathrm{g}}}{\rho_{1}}\right)_{3}}}=\frac{U_{6}}{\sqrt{g D_{6}\left(\frac{\rho_{1}-\rho_{\mathrm{g}}}{\rho_{1}}\right)_{6}}}$

or

$U_{6}=U_{3} \frac{\sqrt{D_{6}\left(\frac{\rho_{1}-\rho_{\mathrm{g}}}{\rho_{1}}\right)_{6}}}{\sqrt{D_{3}\left(\frac{\rho_{1}-\rho_{\mathrm{g}}}{\rho_{1}}\right)_{3}}}$

Thus for a pipe diameter larger than that of the pipe used in the tests, for example, a 6-inch pipe, and for fluids other than low density air (in all of our tests $\rho<2 \mathrm{~kg} / \mathrm{m}^{3}$ ), where the density ratio is much less than unity, we might expect to observe a kill velocity approximately

$U_{6} \approx U_{3} \sqrt{\frac{D_{6}}{D_{3}}} \sqrt{\left(\frac{\rho_{1}-\rho_{\mathrm{g}}}{\rho_{1}}\right)_{6}}$,

where the $U_{3}$ velocity is that predicted by the curves given earlier, i.e. $U_{\text {water }}$ or $U_{\text {brine }}$.

As discussed in Levy (1999), a study by Ellis and Jones suggests that the scaling effect diminishes in pipes larger than about 6 in., since gas slugs typically break up in larger pipes and yield a churn flow. Thus, one might expect that the kill velocity will reach a maximum under these conditions and, therefore, not exceed much more than that predicted above. Based on our present experiments, we find that liquid gas kill velocity varies between 1.2 and $1.4 \mathrm{~m} / \mathrm{s}$ for water and 1.0 and
$1.2 \mathrm{~m} / \mathrm{s}$ for brine, based on the variable gas flow rates in a vertical pipe. This Froude scaling places our measurements in a range of $1.73-2.02 \mathrm{~m} / \mathrm{s}$ for water and $1.44-1.74 \mathrm{~m} / \mathrm{s}$ for Brine, in a larger pipe (for the range of gas flows we tested). Furthermore, if one considers the density ratio, which will be less than unity for larger density highly compressed gas flows, one will see a 10-15\% reduction in these figures. Industry best practice seems to indicate that a successful kill can be achieved with a liquid velocity of around $2 \mathrm{~m} / \mathrm{s}$, which agrees well with our water and brine measurements.

Reservoir scenario analysis

Based on reservoir data for an ideal flowing gas well, i.e. 37,000 standard $\mathrm{m}^{3} / \mathrm{h}\left(10.2\right.$ standard $\left.\mathrm{m}^{3} / \mathrm{s}\right)$ at $28 \mathrm{MPa}$ and $96{ }^{\circ} \mathrm{C}$, we find that the actual gas flow yields approximately 0.04747 Actual $\mathrm{m}^{3} / \mathrm{s}$, or for a $61 / 4 \mathrm{in}$. ID bore a gas velocity of $2.39 \mathrm{~m} / \mathrm{s}$. In the lab simulations of dynamic well kill, the actual gas flow velocity varied between 1 and $5 \mathrm{~m} / \mathrm{s}$ with a liquid kill flow velocity of approximately $1.2-1.4 \mathrm{~m} / \mathrm{s}$ for water and $1.0-1.2 \mathrm{~m} / \mathrm{s}$ for the brine mixture in the vertical pipe. For brine, we find at $2.4 \mathrm{~m} / \mathrm{s}$ gas velocity a kill velocity of approximately $1.1 \mathrm{~m} / \mathrm{s}$. Further given the Froude number scaling for larger pipes, one may expect an increase by a factor of 1.44 for a 7 -in. OD well bore with a $6 \frac{1}{4}-$-in. ID, yielding a predicted kill velocity of $1.59 \mathrm{~m} / \mathrm{s}$, when no density effect is considered, in the absence of actual $p-v-T$ data for the reservoir gas.

Since a density correction factor will be less than unity yielding a brine kill velocity of less than $1.59 \mathrm{~m} / \mathrm{s}$. Table 1 shows kill velocities for some selected ideal gases when density effects are considered. Thus, a brine kill for this flowing well scenario might require a superficial velocity of approximately $1.4-1.5 \mathrm{~m} / \mathrm{s}$ for a typical ideal gas. A proper equation of state should be utilized for a real reservoir gas, when computing gas density correction factor. The brine density was assumed to be $1,250 \mathrm{~kg} / \mathrm{m}^{3}$.

Drift flux modeling

Griffith and Wallis (see Levy 1999) proposed that effective gas bubble rise velocity in a two-phase flow varies with a homogeneous mixture velocity and the static bubble rise according to

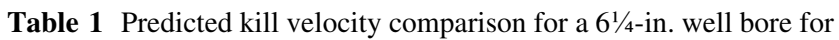
brine in a vertical pipe

\begin{tabular}{llll}
\hline Gas & Flow $\left(\mathrm{m}^{3} / \mathrm{s}\right)$ & $\begin{array}{l}\text { Actual gas } \\
\text { velocity }(\mathrm{m} / \mathrm{s})\end{array}$ & $\begin{array}{l}\text { Kill velocity } \\
(\mathrm{m} / \mathrm{s})\end{array}$ \\
\hline Air & 0.04747 & 2.39 & 1.41 \\
Methane & 0.04747 & 2.39 & 1.49 \\
Ethane & 0.04747 & 2.39 & 1.41 \\
\hline
\end{tabular}




$$
U_{\mathrm{BRV}}=\left(U_{\mathrm{g}} \mp U_{\mathrm{l}}\right)+C_{0} \cdot(0.345 \sqrt{g D})
$$

This expression accounts for the combined effect of the mixture flow whether concurrent or countercurrent and the added effect of an increased axis velocity on bubble rise, i.e. the constant $C_{0}$.

According to Nicklin (see Levy 1999), the actual bubble rise velocity will depend on

$$
U_{\mathrm{BRV}}=1.2\left(U_{\mathrm{g}} \mp U_{\mathrm{l}}\right)+0.345 \sqrt{g D}
$$

which indicates that a small bubble will rise with a speed related to the net two-phase flow velocity (first term) plus the static bubble rise velocity.

In either case, if the liquid and gas are flowing concurrently, it will increase the effective bubble rise velocity, or if the flow is countercurrent, then the bubble rise will be retarded by this flow. The correct form is a matter of debate, which should be connected to the size of the actual bubble and the bubble Reynolds number.

While strictly valid only for a single bubble in a liquid column, we can use the above approach as a modeling tool for a continuous gas stream. Using the above as a guide, in a countercurrent flow containing a steady stream of gas slugs, if we wish to hold stationary this flow then we may re-arrange the above equations to give a zero effective BRV and approximately obtain:

$0=\left(U_{\mathrm{g}}-U_{\mathrm{l}}\right)+0.345 \sqrt{g D}$

or

$U_{1}=U_{\mathrm{g}}+C \cdot 0.345 \sqrt{g D}$,

where $C$ is given a nominal value $C \sim 1$, as it is of this order of magnitude. A more general form of the above equations with two arbitrary constants has been proposed by others (see Levy 1999), as a means of modeling a two phase slug flow:

$U_{\mathrm{BRV}}=C_{\mathrm{l}}\left(U_{\mathrm{g}} \mp U_{1}\right)+C_{2} \cdot \sqrt{g D\left(\frac{\rho_{1}-\rho_{\mathrm{g}}}{\rho_{\mathrm{l}}}\right)}$

This shows that the effective liquid kill velocity is connected to the static bubble rise and the net superficial gas flow (gas flowing alone in the pipe). The constant $C_{1}$ is related to the homogeneous velocity flow, i.e. $C_{1} \sim 1.2$ for turbulent flows, and $C_{1} \sim 2$ for laminar flows, while $C_{2}$ is related to surface tension, inertial, and viscosity effects as discussed before.

Based on the present results, we can propose a simple drift flux based model using the following:

$U_{\mathrm{l}}=U_{\mathrm{g}}+\frac{C_{2}}{C_{1}} \cdot \sqrt{g D\left(\frac{\rho_{1}-\rho_{\mathrm{g}}}{\rho_{\mathrm{l}}}\right)}$,

where the constant $C_{2}$ is that obtained from our present experiments for static BRV in brine or water. For a vertical pipe, we measured $C_{2}=0.4455$ for water and $C_{2}=0.3251$ for brine mixture. For a dynamic test, we could use the above in the following form for future studies:

$U_{\mathrm{l}}=U_{\mathrm{g}}+C_{0} \cdot \sqrt{g D\left(\frac{\rho_{1}-\rho_{\mathrm{g}}}{\rho_{1}}\right)}$

The constant $C_{0}$ is now a function of the properties of the kill fluid and pipe inclination as discussed earlier.

As an example, if one considers the present experiments, a dynamic kill using approximately $5.85 \mathrm{~L} / \mathrm{s}$ of water at 50 SCFM should be considered. During most of our experiments, the pressure of the gas was approximately $18 \mathrm{psi}$ and had a temperature of approximately $20^{\circ} \mathrm{C}$. This yields a gas density of approximately $2.68 \mathrm{~kg} / \mathrm{m}^{3}$. The actual superficial gas velocity that we would obtain at 50 SCFM under these conditions is approximately $2.35 \mathrm{~m} / \mathrm{s}$. The $5.85 \mathrm{~L} / \mathrm{s}$ of water yields a superficial water velocity of approximately $1.276 \mathrm{~m} / \mathrm{s}$. If we use the Griffith and Wallis form given above we find that

$U_{1}=2.35+0.345 \sqrt{9.81 \times 0.076} \approx 2.65$,

which is approximately twice what was measured. This gap widens at larger flows, which leads one to question whether the single slug flow drift flux model should be applied to a continuous stream of gas. However, Eq. (33) may still have value in future tests as a single constant is all that is required for a given kill fluid.

\section{Conclusions}

Dynamic well kill flow measurements were performed. The data obtained support observed field results and also concur with static bubble rise results published in the literature.

Dynamic gas kill flow rates were shown to scale in a similar manner as the static bubble rise data. The scaled data for liquid kill velocities appear to support the field data.

The simple empirical models developed in this paper are most reliable for the test fluids considered, i.e. water and $30 \%$ brine mixture. These results cover standard gas flow rates in the range of 25-100 SCFM of air, which yielded actual gas phase velocities of $1-5 \mathrm{~m} / \mathrm{s}$ during our tests.

The effects of surface tension, fluid viscosity, and fluid density need further examination, particularly for nonNewtonian fluids such as the Xanvis shear thinning fluid.

The effect of pipe diameter should also be considered further as Froude number scaling has been questioned above 6 in. pipe diameters. All of our tests only considered the flow in a nominal 3 in. pipe. At least one additional pipe diameter, i.e. a nominal 6 in. ID, should be considered 
for future studies. This will allow the Froude number scaling to be adequately verified for the dynamic kill tests.

Additionally, a third type of test, which is actually a combination of the static and dynamic measurements, would also yield useful data. This test would release a single slug of gas similar to the static tests, but rather than a static liquid column, a liquid flow of known flow rate would be imposed against the rising slug.

Finally, actual $p-v-T$ behavior for reservoir gases needs to be considered when determining the well bore gas density used in the scaling calculations. This aspect provides the greatest uncertainty in the present analysis when considering the kill velocity in a 6 -in. well bore in the reservoir scenario analysis.

Open Access This article is distributed under the terms of the Creative Commons Attribution License which permits any use, distribution, and reproduction in any medium, provided the original author(s) and the source are credited.

\section{References}

Adams NJ, Hansen A, Stone AD, Voism JA (2008) A case history of underwater wild well capping: successful implementation of new technology on the SLB-5-4X blowout in Lake Maracaibo Venezuela. SPE Asia Pacific oil \& gas conference and exhibition, Dallas TX, Sep, pp 27-30

Agarwal A, Tai CF, Chung JN (2010) Unsteady development of a deformable bubble rising in quiescent liquid. J Comput Meth Appl Mech Eng 199:1080-1090

Bendiksen KH (1985) On the motion of long bubbles in vertical tubes. Int J Multiph Flow 11:797-812

Berghmans J (1973) Stability of gas bubbles rising in inviscid fluids. J Chem Eng Sci 28(11):2005-2011

Bugg JD, Mack K, Rezkhallah KS (1998) A numerical model of Taylor bubble rising through stagnant liquids in vertical tubes. Int J Multiph Flow 24(2):271-28

Carew PS, Thomas NH, Johnson AB (1995) A physically based correlation for the effects of power law rheology and inclination on slug bubble rise velocity. Int J Multiph Flow 21(6):1091-1106

Conde M (2009) Aqeous solutions of lithium and calcium chlorides: property formulations for use in air conditioning equipment design. M. Conde engineering, technical report
Dziubinski M, Orczkowaska M, Budzynski P (2003) Comment on bubble rising velocity in non-newtonian liquid. J Chem Eng Sci 58:2441-2443

Fabre J, Line A (1992) Modeling of two-phase flow. Annu Rev Fluid Mech 24:21-46

Fram JW (1994) Well kill (quenching) study of the thermal producers in the South Belridge field. SPE Production \& Facilities, California

Han W, Zhen-Yu Z, Yong-Ming Y, Hui-Sheng Z (2010) Viscosity effects on the behavior of a rising bubble. J Hydrodyn 22:81-89

Hua J, Stene J, Lin P (2008) Numerical simulation of 3D bubble rising in viscous liquid using a front tracking method. J Comput Phys 227:3358-3382

Hua J, Shuhau W, Jing W (2009) Experimental study of motion of nitrogen Taylor bubbles and liquid slugs in inclined tubes. Chin J Aeronaut 22:349-354

Kastor RL, Letbetter SC (1974) Extra increments of pressure or mud weight safety factors added during well killing procedures can be unsafe. SPE paper 4973

Koedritz WL, Beck FE, Langlinais JP, Bourgoyne AT (2008) Method for determining the feasibility of dynamic kill of shallow gas flows. Presented at the 2008 SPE Asia Pacific Oil \& Gas conference and exhibition, Dallas TX, September 27-30

Levy S (1999) Two phase flow in complex systems. Wiley, New York

Liao Q, Zhao TS (2003) Modeling of Taylor bubble rising in a vertical mini noncircular channel filled with a stagnant liquid. Int J Multiph Flow 29:411-434

Rubiandini RS (2008) Dynamic killing parameters design in underground blowout. SPE Asia Pacific drilling technology conference and exhibition, Jakarta, Indonesia

Sakakibara K, Yamada M, Miyamoto Y, Saito T (2007) Measurement of the surrounding liquid motion of a single rising bubble using a dual-camera PIV system. J Flow Meas Instrum 18:211-215

Salehi S, Hearland G, Soroush M, Khademi Dehkordi K (2008) Killing of the a gas well: successful implementation of innovative approaches in a Middle-Eastern Carbonate FieldAfield Case. In: Presented at the 2008 SPE Asia Pacific Oil \& Gas conference and exhibition, Perth, Australia

Vallejo-Arrieta VG (2002) Analytical model to control off-bottom blowouts utilizing the concept of simultaneous dynamic seal and bull heading. $\mathrm{PhD}$ dissertation, Louisiana State University, Baton Rouge

Wallis GB (1969) One dimensional two phase flow. McGraw-Hill, New York

Wang Z, Tong AY (2008) Deformation and oscillation of a single gas bubble rising in a narrow vertical tube. Int $J$ Thermal Sci 47:221-228 